\title{
New and extended-action treatments in the management of ADHD: a critical appraisal of lisdexamfetamine in adults and children
}

This article was published in the following Dove Press journal:

Neuropsychiatric Disease and Treatment

15 May 2010

Number of times this article has been viewed

\section{Diana Domnitei \\ Vishal Madaan}

Department of Psychiatry, Creighton University Medical Center,

Omaha, NE, USA
Correspondence: Vishal Madaan Department of Psychiatry, Creighton University Medical Center, 3528, Dodge Street, Omaha, NE 68I 3 I, USA

Tel + I (402) 345-8828

Email vishalmadaan@creighton.edu

\begin{abstract}
Treatment guidelines from the American Academy of Child and Adolescent Psychiatry and the American Academy of Pediatrics state that stimulant medications have the most evidence for safety and efficacy in the treatment of childhood attention deficit hyperactivity disorder (ADHD). Longer-acting stimulants are thus considered as first-line for management of ADHD symptoms. Over the years, concerns about the abuse potential of stimulants have led to the development of alternative formulations of these agents. One such recent development, lisdexamfetamine (LDX) was FDA approved for treating ADHD in children in early 2007 and in adults in early 2008. LDX is a prodrug, which when orally ingested, is converted to l-lysine and active d-amphetamine, which is responsible for its therapeutic activity. This unique formulation may lead to a possible reduction of the abuse potential, by bypassing the first-pass metabolism. In fact, a statistically significant difference for the 'liking' effects on the Drug Questionnaire Response has been reported with intravenous LDX compared to d-amphetamine. LDX appears to have an efficacy and tolerability profile comparable to other extended-release stimulant formulations used to treat $\mathrm{ADHD}$, but reduced potential for abuse-related liking effects when compared to equivalent amounts of immediate-release d-amphetamine. The most common adverse events include decreased appetite, insomnia, upper abdominal pain, headache, irritability, weight loss, and nausea.
\end{abstract}

Keywords: lisdexamfetamine, attention deficit hyperactivity disorder, adults, children

\section{Introduction}

Treatment guidelines from the American Academy of Child and Adolescent Psychiatry and the American Academy of Pediatrics state that stimulant medications have the most evidence for safety and efficacy in the treatment of childhood attention deficit hyperactivity disorder (ADHD). Longer-acting stimulants are thus considered as first line for management of ADHD symptoms. ${ }^{1,2}$ Over the years, concerns related to the abuse potential of stimulants have led to the development of alternative formulations of these agents. ${ }^{3}$

One such recent development, lisdexamfetamine (LDX) was approved by the Food and Drug Administration (FDA) for treating ADHD in children in early 2007 and in adults in early 2008. LDX is a prodrug, which when orally ingested, is converted to 1-lysine and active d-amphetamine, which is responsible for its therapeutic activity. This unique formulation may lead to a possible reduction of the abuse potential, by bypassing the first-pass metabolism. In fact, a statistically significant difference for the 'liking' effects on the Drug Questionnaire Response, has been reported with intravenous LDX compared to d-amphetamine. ${ }^{4}$ 
ADHD is a common childhood neurobehavioral disorder with an estimated prevalence of $7 \%$ to $10 \%$ in school-aged children. ${ }^{5,6}$ Childhood ADHD presents with hyperactivityimpulsivity and/or inattentive behaviors which occur more commonly and are of a greater severity than would normally be seen in children of similar age and developmental level. According to the National Comorbidity Survey, symptoms of ADHD persist into adolescence and adulthood severely impairing function in $37 \%$ to $85 \%$ of those affected. ${ }^{6}$

Stimulants are regarded as first-line pharmacotherapy in children and adults and are currently the most widely prescribed medications for the treatment of ADHD. ${ }^{7,8}$ There is evidence to suggest that the lack of consistency and predictability in their bioavailability may result in variable efficacy and duration of effect. ${ }^{9-11}$ While these medications are effective and largely safe, they may mediate reinforcing effects, which has often led to concerns about abuse potential. ${ }^{12}$ Unmet therapeutic needs in ADHD include having a consistent delivery of the medication, adequate duration of action, and also reduced potential for abuse.

LDX is the first long-acting prodrug stimulant to be developed and provides a long acting duration of effect that is consistent throughout the day, with a potential for less abuse compared to equivalent amounts of immediate-release d-amphetamine. ${ }^{13,14}$ Prodrugs are inactive compounds that require in vivo conversion to release therapeutically active medications. Children 6 to 12 years of age who were treated with LDX showed improvement of ADHD symptoms as early as the first week of treatment, with tolerance of side effects in short-term, placebo-controlled studies..$^{10,14}$

LDX is a therapeutically inactive molecule which is converted to 1-lysine, a naturally occurring essential amino acid, and active d-amphetamine after ingestion, the latter of which is responsible for the therapeutic activity. The conversion of LDX to d-amphetamine is not affected by gastrointestinal $\mathrm{pH}$ and is unlikely to be affected by alterations in normal gastrointestinal transit times. ${ }^{16}$ Another pharmacokinetic interaction study indicated a lack of drug interaction with concomitant administration proton pump inhibitor omeprazole with LDX. LDX can thus be co-administered with medications that alter gastrointestinal $\mathrm{pH}$.

LDX was designed to have efficacy and tolerability comparable to other extended-release stimulant formulations used to treat ADHD, but with reduced potential for abuse. It has a chemical designation of (2S)-2,6-diamino-N-[(1S)-1-methyl2-phenylethyl] hexanamide dimethanesulfonate and the corresponding molecular formula is $\mathrm{C}_{15} \mathrm{H}_{25} \mathrm{~N}_{3} \mathrm{O} \bullet\left(\mathrm{CH}_{4} \mathrm{O}_{3} \mathrm{~S}_{2}\right)$. LDX has a molecular weight of 455.60. LDX is formulated as a white water soluble powder and is only available in capsules, containing $20 \mathrm{mg}, 30 \mathrm{mg}, 40 \mathrm{mg}, 50 \mathrm{mg}, 60 \mathrm{mg}$, and $70 \mathrm{mg}$ of LDX ${ }^{17} \mathrm{LDX}$ consists of d-amphetamine covalently attached to an essential amino acid, L-lysine.

\section{Mechanism of action}

LDX is a prodrug which is pharmacologically inactive and undergoes biotransformation before exhibiting its pharmacological effects. After oral ingestion, LDX undergoes first pass intestinal and/or hepatic metabolism, the exact mechanism of which has not yet been elucidated. It is quickly absorbed from the gastrointestinal tract and converted to L-lysine and the active drug moiety d-amphetamine via enzymatic cleavage of the amide linkage. Preclinical studies propose that the systemic circulation is the primary site $(90 \%)$ of this conversion. ${ }^{16,17}$ This is evident from the concentrationtime profile of LDX, which displays a rapid peak plasma concentration ( $\mathrm{T}_{\max } 1$ hour) as well as the simultaneous slow rise of d-amphetamine concentrations, with peak levels of d-amphetamine found 3.5 hours after oral ingestion. Thus the contribution of the gastrointestinal tract to the conversion of LDX to d-amphetamine is low and not affected by gastrointestinal $\mathrm{pH}$ or gastrointestinal transit times. The result is the conversion of LDX to d-amphetamine which mediates its therapeutic effect by blocking norepinephrine and dopamine reuptake into presynaptic neurons and increasing their release into the extraneuronal space.

\section{Pharmacokinetics}

After oral administration, LDX is rapidly absorbed from the gastrointestinal tract and is converted to d-amphetamine and L-lysine. In a Phase I, open label, crossover study where 18 children with ADHD and aged 6 to 12 years, fasted for 8 hours and then were given a single oral dose of 30-, 50- or 70-mg dose of LDX. The $\mathrm{T}_{\max }$ of LDX in this study was found to be 1 hour and the $\mathrm{T}_{\max }$ of $\mathrm{d}$-amphetamine was approximately 3.5 hours. This suggests that the systemic circulation is the site of conversion. ${ }^{17} \mathrm{D}$-amphetamine pharmacokinetics following single-dose oral administration of LDX was found to be linear over the dose range of 30 to $70 \mathrm{mg}$ in 6- to 12-year-old children with no effect observed in the area under the concentration - time curve (AUC).

Data from a Phase I, single-dose, randomized, open-label crossover study in adults administered a single-dose of $70 \mathrm{mg}$ after either fasting, consuming a high fat meal, or ingesting a solution containing capsule contents, resulted in equivalent bioavailabilities based on maximum drug concentration $\left(\mathrm{C}_{\max }\right)$ and AUC. However, the fed patients experienced $\mathrm{T}_{\max }$ 
which was prolonged by 1 hour as compared to the patients who had fasted. ${ }^{18}$

Food does not affect absorption of LDX and delays Cmax by 1 hour or less, therefore LDX can be given with breakfast without affecting therapeutic effect. In contrast, food prolonged the $\mathrm{T}_{\text {max }}$ of $\mathrm{d}$-amphetamine from extendedrelease mixed amphetamine salts (MAS XR) by 2.5 hours compared with the fasted state. ${ }^{19}$ A Phase II, randomized, multi-center, double blind trial found that the $\mathrm{T}_{\text {max }}$ of LDX was 3.5 times less variable than with MAS XR, based on coefficients of variation of $15.33 \%$ for LDX and $52.77 \%$ for MAS XR. Thus patients who took $70 \mathrm{mg}$ of LDX had a more predictable release of $\mathrm{d}$-amphetamine than those who took $30 \mathrm{mg}$ of MAS XR. In addition, the solubility, conversion for LDX to d-amphetamine, and absorption of LDX is not affected by variations in gastric $\mathrm{pH} .{ }^{10}$ Pharmacokinetics of d-amphetamine were found to be similar in pediatric and adolescent ADHD patients and healthy adult volunteers.

It is thought that LDX is not metabolized by cytochrome P450 enzymes, and hence, it does not inhibit the majority of these enzymes. ${ }^{20}$ Krishnan's investigation of the P450 interaction with LDX demonstrated that incubation of LDX with human liver microsomes did not produce concentration-dependent or mechanism-based inhibition of any of the isoenzymes under investigation: CYP1A2, CYP2A6, CYP2B6, CYP2C9, CYP2C19, CYP2D6, and CYP3A4. While active d-amphetamine is finally metabolized to benzoic acid and hippuric acid, results from this study indicate that any drug interactions would likely be caused by amphetamine and its metabolites rather than the intact LDX prodrug. The drug reaches steady-state concentrations in 2 to 3 days. $^{21}$

The recommended initial dose of LDX is $30 \mathrm{mg}$ daily, independent of prior stimulant treatments, the patient's age, and weight. The therapy should be titrated in 10-mg increments at weekly intervals until therapeutic effect is achieved. ${ }^{17}$ It is administered in the morning, to limit the adverse effect on sleep and can be given with or without food. LDX doses of 30,50 , and $70 \mathrm{mg}$ are equivalent in base content of $\mathrm{d}$ amphetamine to extended-release MAS XR doses of 10, 20, and $30 \mathrm{mg}$, respectively. ${ }^{15}$ Doses greater than $70 \mathrm{mg} /$ day have not been studied in children or adults.

\section{Efficacy studies}

Two short-term double-blind, multi-center clinical studies in school aged children who met DSM-IV-TR criteria for ADHD assessed the efficacy and safety profile of once daily oral LDX. ${ }^{10,15}$ The first study by Biederman et al was a phase II multicenter, double blind, placebo and active controlled 3- treatment 3-period crossover study ${ }^{10}$ in 52 children aged 6 to 12 years. The study was conducted in a controlled classroom environment with children with a mean age of 9.1 years (SD 1.7 years) in whom the time from initial ADHD diagnosis was a mean of 3.3 years (SD 2.3 years). Exclusion criteria included comorbid medical and psychiatric disorders which could affect efficacy or tolerability of the stimulants; history of seizures within the past 2 years; significant laboratory abnormalities; history of drug abuse; current use of central nervous system medications; history of allergy to stimulants; and hyperthyroidism, tic disorders, or cardiac disorders. ${ }^{10}$ Inclusion criteria required the capacity to follow classroom instructions, age-appropriate academic functional capacity and a history of adequate response to stable dose stimulant medication of at least 1 month during the previous 6 months.

The subjects in this study initially received MAS-XR for 3 weeks. Based on response as measured on the Clinical Global Impression (CGI) score, interviews with parents and safety data, the doses were either kept the same, or titrated up or downwards by $10-\mathrm{mg}$ increments, with a minimum dose of $10 \mathrm{mg}$ /day. Efforts to compare placebo, MAS XR and LDX, were accomplished by assigning patients to 3 cohorts based on the initial titration phase delineating the optimal dose of MAS XR. The first group received placebo, MAS XR $10 \mathrm{mg} /$ day and LDX $30 \mathrm{mg} /$ day each for 1 week. The second group was given placebo, MAS XR $20 \mathrm{mg} /$ day and LDX $50 \mathrm{mg} /$ day each for 1 week. The third group received placebo, MAS XR $30 \mathrm{mg} /$ day and LDX $70 \mathrm{mg} /$ day each for 1 week. The Swanson, Kotkin, Agler, M-Flynn, and Pelham (SKAMP) scale and Permanent Product Measure of Performance Derived Measures (PERMP) were used as primary efficacy measures to compare improvement in ADHD symptoms among the three agents. This study found that both MAS XR and LDX produced similar improvements, both of which were superior at all doses as compared to placebo. Adverse events were reported in $16 \%$ of LDX patients, $18 \%$ of MAS XR patients, and $15 \%$ of placebo patients. The most common side effects were insomnia $(8 \%)$, decreased appetite (6\%) and anorexia (4\%).

The second study was conducted by Biederman et al. This was a phase III, randomized, placebo-controlled, double-blind trial that included 290 children between the ages of 6 and 12 years, parallel group study (201 boys, 89 girls) diagnosed with either combined or hyperactive subtypes of ADHD. ${ }^{15}$ After a 1-week washout period, these subjects were randomly assigned to received LDX (doses of $30 \mathrm{mg}$ /day, $50 \mathrm{mg}$ /day or $70 \mathrm{mg}$ /day) or placebo for 4 weeks. Groups consisted 
of $94.4 \%, 95.9 \%, 97.3 \%$, and $95.8 \%$ of patients diagnosed with combined type ADHD, respectively. Improvement was measured by change from baseline on the ADHD Rating Scale Version IV (ADHD-RS-IV), which was considered the primary efficacy end point. ADHD-RS IV scores decreased by $50 \%$ to $59 \%$ in all three LDX groups, with the greatest improvement noted in the $70 \mathrm{mg}$ /day group, as compared to a $15 \%$ reduction in the placebo group. Distinctions within each dose group, comparing the improvements in the combined type vs the hyperactive subtype of ADHD were not made in this study, although each group was predominately ADHD combined type. In addition, this study also looked at parental perception of symptom improvement as a secondary efficacy outcome measure using a change from baseline on the Conner's Parent Rating Scale-Revised Short Form (CPRS-R). This scale assessed parental perception of ADHD symptoms of children on LDX and reported that ADHD symptoms were significantly improved at each time point with all LDX daily doses. Another secondary efficacy outcome measure, the CGI-I score, showed $>70 \%$ of patients in the active treatment groups were "very much improved or much improved" at 10 AM, 2 PM, and 6 PM compared with $18 \%$ observed in the placebo group $(P<0.001)$. These results indicate that LDX may be effective throughout the day. ${ }^{15}$

\section{Safety and tolerability}

Efficacy and tolerability profiles in the same phase III study by Biederman et $\mathrm{al}^{15}$ in school-aged children with ADHD was assessed using tolerability assessments which consisted of patient interview and observation, electrocardiogram (ECG), physical examination, and laboratory and biochemical tests. Researchers reported that treatment with LDX was not associated with any significant changes in mean ECG parameters, laboratory values, and systolic and diastolic blood pressures. Adverse events were experienced in $72 \%$ of the children who received LDX $30 \mathrm{mg}, 68 \%$ of children who received $50 \mathrm{mg}$ and $84 \%$ of children who received LDX $60 \mathrm{mg}$. Amongst the placebo group, 47\% of patients reported adverse events. ${ }^{15}$

Over $95 \%$ of the adverse events for all doses of LDX were mild or moderate and consisted of: decreased appetite (39\% of subjects in the LDX group vs $4 \%$ with placebo), insomnia ( $18.8 \%$ vs $3 \%$ ), headache ( $11.9 \%$ vs $10 \%)$, upper abdominal pain ( $11.9 \%$ vs $6 \%$ ), and irritability (10\% vs $0 \%$ ). Most adverse events began during the first week of treatment. No severe adverse events were observed and the majority of all events reported had resolved within 4 weeks of continued therapy.
Although LDX treatment was not associated with significant changes in mean ECG parameters, this study found that children on LDX had a significant increase in mean heart rate when compared to the placebo group. It was also reported that the highest placebo-adjusted mean increase of 4 to 5 beats per minute occurred with the $70-\mathrm{mg}$ dose at endpoint, although no significant differences were reported at each treatment week.

In the phase II multicenter, double blind, placebo and active controlled 3-treatment, 3-period crossover study in a controlled classroom environment by Biederman et $\mathrm{al}^{10}$ three cohorts received LDX, MAS XR, and placebo once daily for 1 week. During the open label part dose titration with MAS XR the adverse events reported were headache (15\%), decreased appetite (14\%), insomnia (10\%), abdominal pain $(6 \%)$, upper respiratory infection $(4 \%)$, affect lability $(4 \%)$, anorexia (2\%), and vomiting (2\%). During the double blind part of the study, the adverse events for LDX, MAS $\mathrm{XR}$ and placebo are reported respectively: decreased appetite $(8 \%, 2 \%, 2 \%)$, decreased appetite $(6 \%, 4 \%, 0 \%)$, upper respirator tract infection $(2 \%, 2 \%, 0 \%)$, vomiting $(0 \%, 2 \%, 4 \%)$, upper abdominal pain $(0 \%, 4 \%, 2 \%)$.

In a poison control review of adverse events to LDX conducted in 5 poison centers spread across 8 states, there were 81 reported cases of LDX ingestion, of which 28 patients or $35 \%$ of those who ingested experienced adverse effects. ${ }^{24}$ Previously reported rates for amphetamines were $3.1 \%$. Of those patients most (79\%) involved first ingestions and $86 \%$ of cases were within the first week of treatment with LDX. The most commonly reported symptoms were agitation (53\%), tachycardia (73\%), dystonia (47\%), insomnia (20\%), hallucinations $(20 \%)$ and chest pain (13\%).

A long-term study of the safety, tolerability and efficacy of LDX was done by Findling et al and consisted of 272 children ages 6 to 12 years who were diagnosed with ADHD by DSM-IV-TR criteria and followed for 12 months. ${ }^{14}$ This study was predominately with white males aged 7 to 11 years diagnosed with ADHD combined type and had participated in prior trials with an average disease duration of 2.3 years. ADHD-RS scores improved by a mean of 27.2 points $(P<0.0001)$ with maintenance of improvements during the first 4 weeks throughout the study. Of the 147 children who completed the study, $>80 \%$ of children at endpoint and $>95 \%$ of completers were rated "improved" based on CGI-I scale.

Over $78 \%$ of children experienced adverse events during the course of the study. These adverse events were considered mild to moderate, and occurred mostly in the first 4 weeks 
of treatment. Over the course of the study, $213(78 \%)$ of 272 treated children experienced a total of 987 treatmentemergent adverse events, of which $97.5 \%$ were mild or moderate in severity. These events occurred during the first 2 months of treatment and after 4 months, with an incidence of $\leq 5 \%$ by 4 months. Treatment-emergent adverse events occurring in $>5 \%$ of children included decreased appetite (33\%), headache (18\%), weight loss (18\%), insomnia (17\%), upper abdominal pain (11\%), upper respiratory tract infection $(11 \%)$, irritability $(10 \%)$, nasopharyngitis $(10 \%)$, vomiting (9\%), cough (7\%) and influenza (6\%). Of the 272 children treated with LDX, 25 (9\%) discontinued treatment due to adverse events. Most commonly ( $>1 \%$ ) reasons for discontinuation were irritability, aggressive behavior, and decreased appetite ( $\mathrm{n}=3$ each; $1.1 \%$ ).

This is the only long-term safety and tolerability study with LDX in children with ADHD. It did not use an objective assessment tool in recording adverse events but instead relied on observation for gathering of adverse event data, thus leading to a potential underreporting of adverse events. Furthermore, most of the patients in this study had received LDX in a previous trial. Thus it is possible that those who experienced acute adverse events in the previous study did not subsequently chose to continue into the current study and data regarding their adverse events are not included in this open label study. This would lead to a lower rate of adverse events reported. In addition, due to the majority of patients being male (69\%) and white (53\%), more trials looking at the relative benefit of LDX in female patients and patients with different ethnic backgrounds, as well as those with comorbid psychiatric illnesses are needed.

There may be unknown long-term complications with using LDX as with other stimulants, and clinicians should utilize the patient's medical history, family history, ECG, blood pressure and pulse as well as a thorough physical examination to assess the presence of cardiovascular disease prior to prescribing stimulants. In August 2008, the American Academy of Pediatrics (AAP) issued a recommendation stating that routine use of ECGs in children receiving medications for ADHD is not warranted. A joint statement by AAP and the American Heart Association states that ECGs are not mandatory, but should be performed at the physician's discretion. $^{25}$

The warning section of the prescribing information includes many of the same precautions as other stimulants used to treat ADHD. LDX has a black box warning pointing out the possibility of abuse of amphetamines, the possibility of drug dependence, and the potential for sudden cardiac death and adverse events when misused. It also warns against the use of LDX and other stimulants in patients with advanced atherosclerosis, structural cardiac abnormalities, moderatesevere hypertension, hyperthyroidism, glaucoma, history of agitation, and patients with a history of drug abuse. ${ }^{17}$

\section{Abuse potential}

LDX is categorized as a Schedule II Controlled Substance according to the United States Drug Enforcement Agency. To date, there are two placebo controlled clinical studies of the oral abuse liability of $\operatorname{LDX}^{22-23}$ and 1 placebo controlled trial of IV abuse liability, ${ }^{4}$ all of them performed using healthy adult subjects with a diagnosis of stimulant abuse based on DSM-IV-TR criteria. Results of all three studies report a lower abuse potential of LDX when compared to immediate-release d-amphetamine sulfate.

One of the initial studies of abuse liability of oral LDX was a double-blind, placebo- and active-controlled study with a single-dose crossover design which evaluated the safety, tolerability and abuse liability of intravenously administered LDX in group of 12 healthy adult volunteers who met criteria of stimulant abuse by DSM-IV-TR at the time of the study. Subjects in this study received single oral doses of 30 to $150 \mathrm{mg}$ LDX, placebo, or immediate release d-amphetamine sulfate in equivalent doses. LDX at equivalent or higher in d-amphetamine content caused less euphoria and drug liking, with a later mean peak effect relative to the d-amphetamine as measured on the Drug Rating Questionnaire-Subjects (DRQS) and the Drug Rating Questionnaire-Observer (DRQO). Subjects reported euphoria within 15 minutes of d-amphetamine, whereas subjects receiving LDX did not show a statistically significant difference compared to placebo in liking effects.

Another abuse liability study by Jasinski et $\mathrm{al}^{23}$ was a double-blind, randomized, placebo- and active-controlled, crossover study. This study was designed to evaluate the likeability, safety and abuse liability of oral LDX in healthy adult volunteers with a DSM-IV-TR diagnosis of stimulant abuse at the time of the study. It enrolled 36 adult (30 male and 6 female) stimulant abusers and compared onset and liking effects of LDX $50 \mathrm{mg}, 100 \mathrm{mg}$, and $150 \mathrm{mg}$ as compared to d-amphetamine $40 \mathrm{mg}$ (equivalent to LDX $100 \mathrm{mg}$ ) and placebo. The study findings indicated that LDX attenuates the onset and intensity of subjective drug liking effects as measured by the DRQS. Furthermore, the liking effects of LDX $\leq 100 \mathrm{mg}$ were not significantly different from placebo. At doses of LDX $150 \mathrm{mg}$, the liking effects were greater than placebo and similar to d-amphetamine $40 \mathrm{mg}$. This may be 
in part due to the mean peak effect of the liking effects in LDX which was delayed as compared to d-amphetamine. The attenuated liking scale scores suggest that LDX may be less reinforcing than an equivalent dose of d-amphetamine.

The abuse liability of IV LDX $25 \mathrm{mg}$ and $50 \mathrm{mg}$ was compared with IV d-amphetamine sulfate $10 \mathrm{mg}$ or $20 \mathrm{mg}$, and with placebo in 12 healthy, non-ADHD volunteers with a diagnosis of stimulant abuse based on DSM-IV-TR criteria in a randomized, single-center, double-blind, 3-way crossover study. Subjects receiving IV LDX $25 \mathrm{mg}$ reported no amphetamine-like effects, and those receiving LDX $50 \mathrm{mg}$ did not report drug liking scores comparable with placebo. D-amphetamine sulfate $20 \mathrm{mg}$ displayed significant drugliking scores. In this study, $89 \%$ of subjects reported that they would not choose to take LDX again. To date, there are no studies studying the oral or IV LDX abuse liability in healthy adults without a diagnosis of stimulant abuse by DSM-IV-TR. ${ }^{4}$

\section{Contraindications and use in special populations}

LDX should be used with caution in patients with known hypersensitivity or idiosyncratic reactions to sympathomimetic amines, hyperthyroidism, glaucoma, moderate to severe hypertension, advanced arteriosclerosis, and patients with a history of drug abuse. LDX is contraindicated for 14 days after discontinuation of monoamine oxidase inhibitor use. LDX has not been studied humans in pregnancy and is a Pregnancy Category C. LDX should be used very cautiously in pregnancy, and only in situations where the benefit outweighs the risk to the fetus as currently no adequate and well-controlled studies in pregnant women have been performed. To date, there has been one report of severe congenital bony deformity, tracheo-esophageal fistula, and anal atresia (VATER association) in a baby born to a woman taking LDX with lovastatin during the first trimester of pregnancy. The package insert for LDX states that while it has not been studied in pregnant women amphetamines are secreted in breast milk. Pregnant females are at increased risk of premature delivery, giving birth to infants with low birth weights, and may give birth to infants with neonatal withdrawal related dysphoria and agitation. ${ }^{17}$ LDX has not been studied in the geriatric population.

\section{Conclusions}

LDX is the first FDA approved long acting prodrug amphetamine stimulant in children aged 6 to 12 years and adults with ADHD. It consists of a moiety of L-lysine and d-amphetamine which requires proteolytic cleavage before the active d-amphetamine is released. It has similar efficacy and tolerability but less potential for abuse than equivalent amounts of immediate release dextroamphetamine. Abuse potential of LDX appears attenuated by the prodrug structure, slow-release formulation, longer $\mathrm{T}_{\max }$, and a more consistent effect throughout the day. LDX has been shown to be less likable than immediate release d-amphetamine based on drug liking effects as measured by the Drug Rating Questionnaire-Subjects and the Drug Rating QuestionnaireObserver in studies of LDX. Therefore, LDX may be part of a comprehensive treatment plan for ADHD which includes educational, psychological, and social interventions.

\section{Disclosures}

Dr Madaan is Director, Child and Adolescent Psychopharmacology, Department of Psychiatry, Creighton University Medical Center, Omaha, NE and has no disclosures to make. Dr Domnitei is senior house officer at Creighton-Nebraska Psychiatry Program, Omaha, NE and has no disclosures to make.

\section{References}

1. Pliszka S, for the AACAP Work Group on Quality Issues. Practice parameter for the assessment and treatment of children and adolescents with attention-deficit/hyperactivity disorder. $J$ Am Acad Child Adolesc Psychiatry. 2007;35:180-184.

2. American Academy of Pediatrics, Subcommittee on Attention-DeficitHyperactivity Disorder Committee on Quality Improvement. Clinical Practice guideline Treatment of the school-age child with attentiondeficit/hyperactivity disorder. Pediatrics. 2001;108:1033-1044.

3. Lopez FA. ADHD: New Pharmacological treatments on the horizon. J Dev Behav Pediatr. 2006;27:410-416.

4. Jasinski DR, Krishnan S. Human pharmacology of intravenous lisdexamfetamine dimesylate: abuse liability in adult stimulant abusers. J Psychopharmacol. 2009;23:410-418.

5. Faraone SV, Sergeant J, Gillberg C, Biederman J. The worldwide prevalence of ADHD: is it an American condition? World Psychiatry. 2003;2:104-113.

6. Kessler RC, Adler L, Barkley R, et al. The prevalence and correlates of adult ADHD in the United States: Results from the National Comorbidity Survey replication. Am J Psychiatry. 2006;163:716-723.

7. Wilens TE, Biederman J, Spencer TJ. Attention deficit/hyperactivity disorder across the lifespan. Annu Rev Med. 2002;53:113-131.

8. Surman CBH, Weisler RH. The state of the art of treatment for pediatric and adolescent ADHD. http://www.medscape.com/viewarticle/ 537383. Accessed 2010 March 31.

9. Connor DF, Steingard RJ. New formulations of stimulants for attention-deficit hyperactivity disorder: therapeutic potential. CNS Drugs. 2004;18:1011-1030.

10. Biederman J, Boellner SW, Childress A, Lopez FA, Krishnan S, Zhang Y. Lisdexamfetamine dimesylate and mixed amphetamine salts extended-release in children with ADHD: a double-blind, placebo-controlled, crossover analog classroom study. Biol Psychiatry. 2007;62:970-976.

11. McGough JJ, Biederman J, Greenhill LL, et al. Pharmacokinetics of SLI381 (ADDERALL XR), an extended-release formulation of Adderall. J Am Acad Child Adolesc Psychiatry. 2003;42:684-691.

12. Elia J, Easley C, Kirkpatrick P. Lisdexamfetamine dimesylate. Nat Rev Drug Discov. 2007;6:343-344. 
13. http://www.fda.gov/ohrms/dockets/ac/08/briefing/2008-4356b1-02Purdue.pdf Accessed 2009 November 7.

14. Findling RL. Evolution of the treatment of attention-deficit/hyperactivity disorder in children: a review. Clin Ther. 2008;30:942-951.

15. Biederman J, Krishnan S, Zhang Y, McGough JJ, Findling RL. Efficacy and tolerability of lisdexamfetamine dimesylate (NRP-104) in children with attention-deficit/hyperactivity disorder: a phase III, multicenter, randomized, double-blind, forced-dose, parallel-group study. Clin Ther. 2007;29:450-463.

16. Najib J. The Efficacy and Safety Profile of Lisdexamfetamine Dimesylate, a Prodrug of d-Amphetamine, for the Treatment of AttentionDeficit/Hyperactivity Disorder in Children and Adults. Clin Ther. 2009;30:142-175.

17. Vyvanse (lisdexamfetamine dimesylate). [package insert] Wayne, PA: Shire US Inc; 2008. http://www.vyvanse.com/index-child.asp. Accessed 2010 March 31.

18. Krishnan S, Zhang Y. Relative bioavailability of lisdexamfetamine 70-mg capsules in fasted and fed healthy adult volunteers and in solution: A single-dose, crossover pharmacokinietic study. J Clin Pharmacol. 2008;48:293-302.

19. Adderall XR mixed amphetamine salts extended release) capsules. [package insert] Wayne, PA: Shire US Inc; 2007.
20. Krishnan S, Moncrief S. An evaluation of the cytochrome p450 inhibition potential of Lisdexamfetamine in liver microsomes. Drug Metab Dispos. 2007;35:180-184.

21. Madaan V. Lisdexamfetamine Dimesylate For Childhood ADHD. Drugs Today. 2008;44:319-324.

22. Jasinski D, Krishnan S. Pharmacokinetics of oral lisdexamfetamine dimesylate (LDX;NRP104) vs d-amphetamine in healthy adults with a history of stimulant abuse. 2006 US Psychiatric and Mental Health Congress; 2006 November 17-19; New Orleans, LA, USA.

23. Jasinski D, Krishnan S. A double-blind, randomized, placebo- and activecontrolled, 6-period crossover study to evaluate the likeability, safety, and abuse potential of lisdexamfetamine dimesylate (LDX) in adult stimulant abusers. 2006 US Psychiatric and Mental Health Congress; 2006 November 17-19; New Orleans, LA, USA.

24. Spiller HA, Griffith JR, Anderson DL, et al. Poison centers detect an unexpectedly frequent number of adverse drug reactions to lisdexamfetamine. Ann Pharmacother. 2008;42:1142-1143.

25. O'Keefe L. ECGs for all ADHD patients? AAP-AHA release joint clarification on AHA recommendation. AAP News. 2008;29:1.
Neuropsychiatric Disease and Treatment

\section{Publish your work in this journal}

Neuropsychiatric Disease and Treatment is an international, peerreviewed journal of clinical therapeutics and pharmacology focusing on concise rapid reporting of clinical or pre-clinical studies on a range of neuropsychiatric and neurological disorders. This journal is indexed on PubMed Central, the 'PsycINFO' database and CAS, and is the official

\section{Dovepress}

journal of The International Neuropsychiatric Association (INA). The manuscript management system is completely online and includes a very quick and fair peer-review system, which is all easy to use. Visit http://www.dovepress.com/testimonials.php to read real quotes from published authors. 